\title{
Active Matter Transport and Jamming on Disordered Landscapes
}

\author{
C. Reichhardt and C. J. Olson Reichhardt \\ Theoretical Division, Los Alamos National Laboratory, Los Alamos, New Mexico 87545, USA
}

(Dated: October 2, 2018)

\begin{abstract}
We numerically examine the transport of active run-and-tumble particles driven with a drift force over random disordered landscapes comprised of fixed obstacles. For increasing run lengths, the net particle transport initially increases before reaching a maximum and decreasing at larger run lengths. The transport reduction is associated with the formation of cluster or living crystal states that become locally jammed or clogged by the obstacles. We also find that the system dynamically jams at lower particle densities when the run length is increased. Our results indicate that there is an optimal activity level for active matter transport through quenched disorder, and could be important for understanding biological transport in complex environments or for applications of active matter particles in random media.
\end{abstract}

PACS numbers: $64.75 . \mathrm{Xc}, 47.63 . \mathrm{Gd}, 87.18 . \mathrm{Hf}$

There has been tremendous growth in interest in what are termed active matter systems, where the individual units comprising the system undergo self-propulsion [13]. Biological examples of such systems include swimming bacteria [4, 5], crawling cells [3], and flocking or swarming particles or agents [6]. Non-biological active matter, such as self-driven colloids [7-10], artificial swimmers [11], and assemblies of self-motile mechanical devices such as bristle bots [12], has been the focus of an increasing number of studies. Active matter systems exhibit a rich variety of behaviors that disappear when the fluctuations experienced by the particles are only equilibrium or thermal in nature. Self-driven particles can exhibit run-and-tumble dynamics similar to that obeyed by many types of bacteria [4, 5] where the particle moves in a fixed direction for a period of time before undergoing a tumbling event and changing directions; alternatively, in the case of active Brownian particles, the particles are driven by a motor in a direction that slowly rotates due to a noise term, a dynamics that appears in many selfdriven colloidal systems [2, 7, 10, 13, 14]. It was recently shown that active Brownian particle dynamics and the run-and-tumble dynamics can be mapped into each other and can be considered equivalent [15].

When collections of repulsively interacting particles such as hard disks undergo thermal fluctuations, they form a uniformly dense state; however, when the particles are active there can be a transition to a phase-separated state consisting of high density clusters residing in a low density active gas [10, 13 20]. In two-dimensional (2D) systems of monodisperse active disks, the clusters have internal hexagonal or crystalline ordering [10, 17, 18]. Recent experiments on light-activated self-driven colloidal particles that form such crystalline cluster states revealed that the clusters are highly dynamical, frequently breaking up and reforming, so that the cluster state has been termed a living crystal [10]. Similar living crystals have also been experimentally observed in other self-driven colloidal systems [18]. The onset of the cluster phase depends both on the density of the system and on the activity level, defined as the distance the particles effectively move in a fixed direction during each run, with cluster phases occurring at lower densities when the activity is increased.

Studies of active matter have generally focused on systems with a smooth substrate. Understanding how active matter particles move in random or complex landscapes is an open question that is relevant to biological systems which, in the wild, typically operate in a complex environment. Possible applications utilizing active particles could also require the particles to interact with disordered landscapes. In studies of active particles interacting with arrays of asymmetric barriers, ratchet effects arise when the activity causes a breaking of detailed balance in the particle-barrier interactions [5, 21 25]. Numerical work has shown that flocking active particles can have their order enhanced by obstacle arrays [26], and that disordered substrates can localize or trap active particles [27].

In this work we focus on the transport of active matter over a disordered substrate in the presence of an external drift force. Particle transport over disordered substrates under an external drift is a very general problem that is studied in a variety of condensed matter systems such as colloids moving over random substrates 28], vortices moving in dirty type-II superconductors [29], sliding charge density waves [30], classical charge transport [31] and the motion of magnetic domain walls [32]. In these systems, the particle transport in the direction of the external drift increases with increasing thermal fluctuations, which diminish the effectiveness of pinning by the substrate. Active particles might be expected to experience significantly reduced pinning effects from the substrate since, as the activity or run length of the particles is increased, the particles would be better able to escape from effective trapping regions; however, in this work we show that for active matter this is generally not the case. We find that an increase in the activity can initially increase the particle transport; however, when 
the run lengths are large enough, the transport becomes strongly impeded due to local jamming effects caused by the formation of living crystals that act like rigid objects, permitting an entire cluster of particles to be pinned by a small number of obstacles. Our results show that there is an optimal run length or activity at which maximum transport of the active matter through disordered media can be achieved. We also demonstrate that the jamming transitions observed in 2D assemblies of non-active disks [33 35] can be connected to active matter systems and that activity can form a new axis of the jamming phase diagram.

Simulation - We consider 2D systems of size $L \times L$ with periodic boundary conditions in the $x$ - and $y$-directions containing an assembly of $N_{a}$ active disks with radius $r_{d}$ that interact with each other via a harmonic repulsion. The dynamics of disk $i$ located at $\mathbf{R}_{i}$ is obtained by integrating the following equation of motion:

$$
\eta \frac{d \mathbf{R}_{i}}{d t}=\mathbf{F}_{i}^{m}+\mathbf{F}_{i}^{s}+\mathbf{F}_{i}^{b}+\mathbf{F}^{D}
$$

where $\eta=1.0$ is the damping constant. Under the motor force $\mathbf{F}_{i}^{m}$, a particle moves in a fixed direction with a constant force $F^{m}$ for a fixed run time $\tau_{r}$, and at the end of each running time a new running direction is chosen at random. We select values of $F_{m}$ such that the particles can never pass through one another or through the obstacles. A single particle in the absence of other particles or obstacles moves a distance $R_{l}=F^{m} \tau_{r}$ in one running time. The steric disk-disk repulsion is modeled as $\mathbf{F}_{i}^{s}=\sum_{i \neq j}^{N_{a}}\left(2 r_{d}-\left|\mathbf{r}_{i j}\right|\right) \Theta\left(2 r_{d}-\left|\mathbf{r}_{i j}\right|\right) \hat{\mathbf{r}}_{i j}$, where $\mathbf{r}_{i j}=\mathbf{R}_{i}-\mathbf{R}_{j}, \hat{\mathbf{r}}_{i j}=\mathbf{r}_{i j} /\left|\mathbf{r}_{i j}\right|$, and we take $r_{d}=0.5$. The term $\mathbf{F}_{i}^{b}$ represents the particle-obstacle interactions, where we model the obstacles as $N_{p}$ immobile disks that have the same steric interactions as the active disks. The disk density $\phi$ is determined by the area covered by all the disks, $\phi=L^{2} / N \pi r_{d}^{2}$, where $N=N_{a}+N_{p}$; here, we consider systems with $L=100$. In the absence of any obstacles or thermal fluctuations, the disks form a hexagonal solid at $\phi \approx 0.9$. The term $\mathbf{F}^{D}=F_{d} \hat{\mathbf{x}}$ represents the external drift force. In order to measure transport we calculate the average drift velocity $\left\langle V_{x}\right\rangle=(1 / N) \sum_{i=1}^{N} \mathbf{v}_{i} \cdot \hat{\mathbf{x}}$. In the absence of a drift force, $\left\langle V_{x}\right\rangle=0$, and in the absence of any obstacles, all the particles move with $\left\langle V_{x}\right\rangle=F_{d}$.

Results - We first characterize the onset of clustering in the absence of quenched disorder. To quantify the amount of clustering present, we utilize a cluster-identification algorithm described in Ref. [36]. In Fig. 1(a-d) we show the onset of cluster formation at a fixed density of $\phi=0.667$ for increasing run lengths $R_{l}$. Different clusters are highlighted by different shadings. At $R_{l}=0.4$ in Fig. 1(a), we find a uniform liquid state, while at $R_{l}=4.0$ in Fig. 1(b), small clusters begin to form. The clusters grow in size and become better defined at $R_{l}=20.0$ in Fig. $1(\mathrm{c})$, and at large $R_{l}$ the
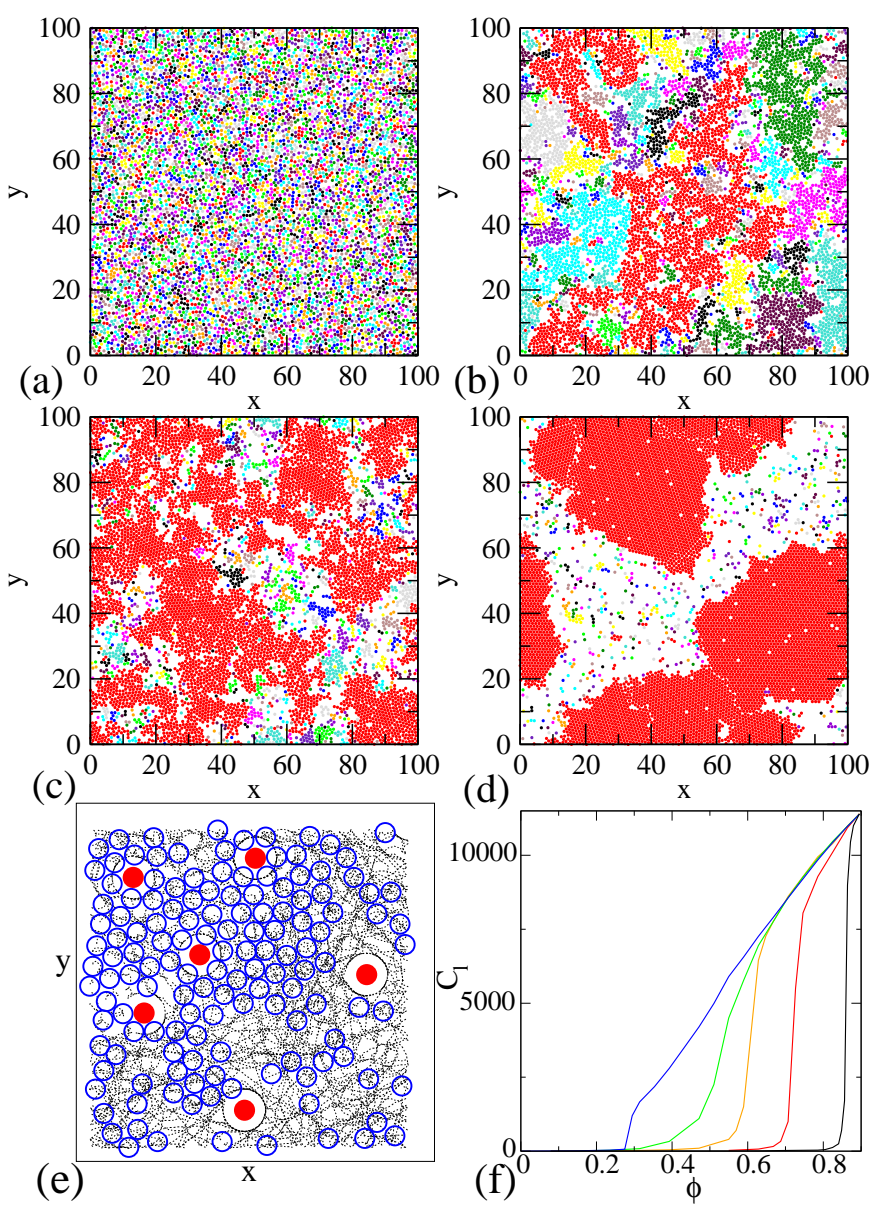

(d)

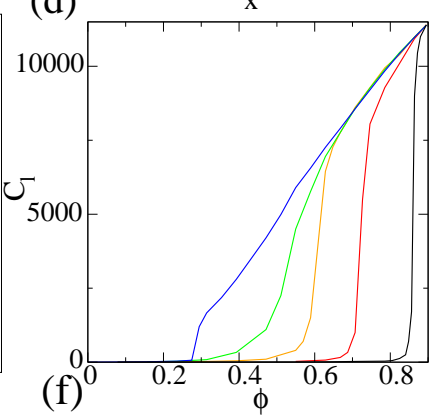

FIG. 1: (a-d) Images of disk positions in an obstacle-free system with $\phi=0.667$ and $N_{p}=0$ for increasing run lengths $R_{l}=$ (a) 0.4, (b) 4.0, (c) 20.0, and (d) 100.0. Individual clusters are indicated with varying shading (coloring), with the largest cluster marked in red. In (d), the system has completely phase separated into a large cluster with local density near $\phi=0.9$ surrounded by a low density gas of particles. (e) Positions of pinned disks (filled circles), active disks (open circles), and disk trajectories over a period of time (dashed lines) in a small section of a sample with $N_{p}=200$ for $R_{l}=20$. (f) $C_{l}$, the number of particles in the largest cluster, vs $\phi$ in the $N_{p}=0$ system for $R_{l}=40,20,1.4,0.2$, and 0.04 , from left to right. The onset of clustering occurs at higher $\phi$ for decreasing run length.

system is completely phase separated into a crystalline hexagonally ordered solid phase with a local density of $\phi=0.9$ surrounded by a low density gas phase, as shown in Fig. 1(d) for $R_{l}=100.0$. This behavior is identical to that found in simulations of active Brownian particles at a fixed density when the persistence length is increased [17]. Cluster formation also occurs when $\phi$ is increased for fixed $R_{l}$. In Fig. 1(f) we plot $C_{l}$, the average number of particles in the largest cluster, versus $\phi$ for systems with $R_{l}=40,20,1.4,0.2$, and 0.04 . At the smallest run length of $R_{l}=0.04$, clustering does not occur until the 

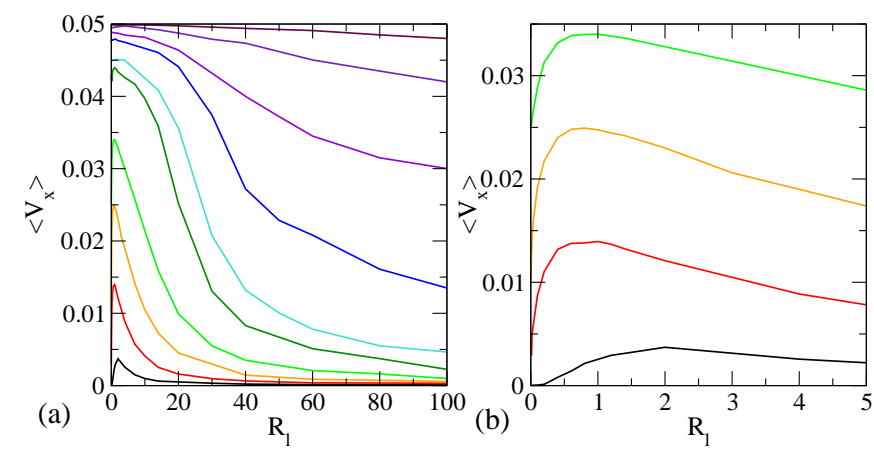

FIG. 2: (a) $\left\langle V_{x}\right\rangle$, the average velocity per particle in the drift direction, vs $R_{l}$ for a system with $\phi=0.667$ and $F_{d}=0.05$. The obstacle density $\phi_{p}=0.00039,0.00157,0.00472,0.0785$, $0.0157,0.02356,0.055,0.0942,0.01413$, and 0.188 , from top to bottom. When $R_{l}>5.0,\left\langle V_{x}\right\rangle$ decreases with increasing $R_{l}$. (b) $\left\langle V_{x}\right\rangle$ vs $R_{l}$ passes through a maximum for all the curves, as shown more clearly in this blow-up of panel (a) for $\phi_{p}=0.055,0.094,0.1413$, and 0.188, from top to bottom.

density is well above $\phi=0.8$, while for large $R_{l}$ the onset of clustering shifts to much lower values of $\phi$.

We next consider the effect of adding $N_{p}$ obstacles in the form of immobile disks, as illustrated in Fig. 1(e). We report both the total density $\phi$ of the system which includes both the active and stationary disks, as well as the density of only the stationary disks $\phi_{p}=L^{2} / N_{p} \pi r_{d}^{2}$. All the active disks experience a uniform drift force with $F_{d}=0.05$, so that in the absence of any obstacles $\left\langle V_{x}\right\rangle=F_{d}=0.05$ independent of the value of $R_{l}$. In Fig. 2(a) we plot $\left\langle V_{x}\right\rangle$ versus $R_{l}$ at $\phi=0.667$ for varied $\phi_{p}=0.00039,0.00157,0.00472,0.0785,0.0157,0.02356$, $0.055,0.0942,0.1413$, and 0.188 . For $R_{l}>5.0$ the particle drift velocity decreases with increasing $R_{l}$. Additionally, increasing the density of obstacles lowers the drift velocity. In each case, $\left\langle V_{x}\right\rangle$ passes through a local maximum as a function of $R_{l}$, as shown more clearly in Fig. 2(b) where the $\left\langle V_{x}\right\rangle$ vs $R_{l}$ curves for $\phi_{p}=0.055$, $0.094,0.1413$, and 0.188 peak around $R_{l}=1.0$. For very short run lengths $R_{l}<0.1$ for the largest obstacle density of $\phi_{p}=0.188$, the system jams and $\left\langle V_{x}\right\rangle \approx 0$. The initial increase in $\left\langle V_{x}\right\rangle$ with increasing $R_{l}$ has the same form that would appear if increasing thermal fluctuations were added to the system: $\left\langle V_{x}\right\rangle=A \exp \left(U / k_{B} T\right)$, where $A$ is the driving force. In the case of thermal fluctuations, the drift velocity would saturate at large $T$ when the substrate loses its effectiveness. In the active matter system for small $R_{l}$, increasing $R_{l}$ is is similar to increasing the temperature; however, once $R_{l}$ is large enough for clustering to occur, the particle density becomes inhomogeneous and $\left\langle V_{x}\right\rangle$ drops. We have examined a variety of values of $\phi$ and $\phi_{p}$ and find that in general, for $\phi>0.4$, when quenched disorder is present the drift velocity is maximized at an optimal value of $R_{l}$.

To show how the onset of clustering correlates with
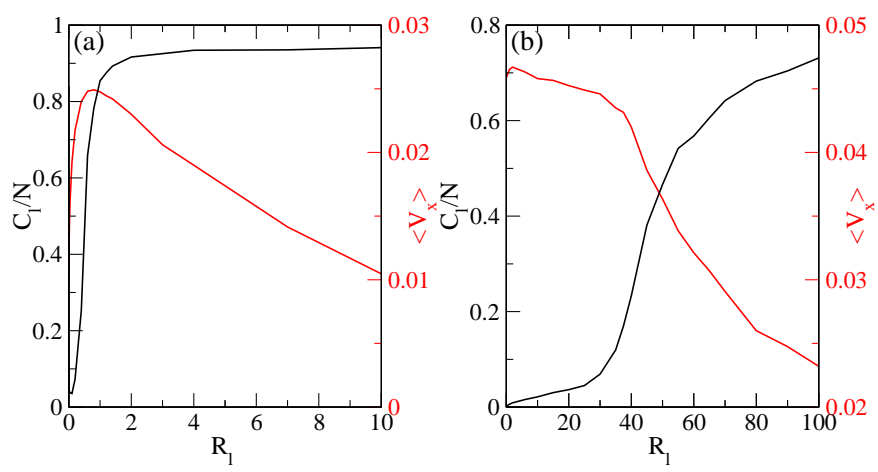

FIG. 3: (a) $C_{l} / N$, the fraction of particles in the largest cluster (dark curve), and $\left\langle V_{x}\right\rangle$ (light curve) vs $R_{l}$ for a system with $\phi=0.667$ and $\phi_{p}=0.094$. The drop in $\left\langle V_{x}\right\rangle$ is correlated with the formation of cluster states. (b) The same for a system with $\phi=0.337$ and $\phi_{p}=0.02356$, where the drop in $\left\langle V_{x}\right\rangle$ also correlates with the onset of clustering.

the decrease in transport, in Fig. 3(a) we plot $\left\langle V_{x}\right\rangle$ and $C_{l} / N$, the fraction of particles in the largest cluster, versus $R_{l}$ for a system with $\phi=0.667$ and $\phi_{p}=0.094$. The decrease in $\left\langle V_{x}\right\rangle$ coincides with an increase in $C_{l} / N$, indicating the formation of clusters. When $R_{l}$ is small, all of the mobile particles are in the gas phase, and if a particle encounters an obstacle, it has a high probability of quickly changing its swimming direction and moving away from the obstacle. As $R_{l}$ increases and the system enters the cluster phase, an obstacle can pin a cluster even if it contacts the obstacle only at a single point, since the cluster has transient rigidity. As a result, the moving clusters can become clogged by the obstacles, reducing the transport. Since the clusters are dynamic, the particles that belonged to a cluster become unpinned once the cluster breaks apart. The lifetime of individual clusters increases with increasing $R_{l}$ and the longerlived clusters remain clogged for longer periods of time, resulting in a gradual reduction in $\left\langle V_{x}\right\rangle$ with increasing $R_{l}$ as shown in Fig. 2. For $\phi_{p}>0.0785$, in the cluster regime where $\left\langle V_{x}\right\rangle$ decreases with increasing $R_{l}$ we find $V_{x}\left(R_{L}\right) \propto R_{L}^{\alpha}$, with $\alpha=1.31 \pm 0.02$ [37]. In Fig. 3(b) we plot $C_{l} / N$ and $\left\langle V_{x}\right\rangle$ vs $R_{l}$ for a system with $\phi=0.337$ and $\phi_{p}=0.02356$. The transport is almost constant at the values of $R_{l}$ where clustering is absent; however, at the onset of clustering where $C_{l} / N$ increases, $\left\langle V_{x}\right\rangle$ begins to drop rapidly, showing the correlation between the clustering and the drop in the transport.

We next show how the transport is affected by the particle density and jamming effects. The jamming concept was originally applied to non-active particles in the zerotemperature limit, where it was shown that as a function of increasing density the system becomes jammed at $\phi_{j}$, termed point $\mathrm{J} 33$. In $2 \mathrm{D}$ assemblies of bidisperse disks, point $\mathrm{J}$ falls at $\phi_{j}=0.844$ [34], while for monodisperse disk assemblies the system forms a hexag- 

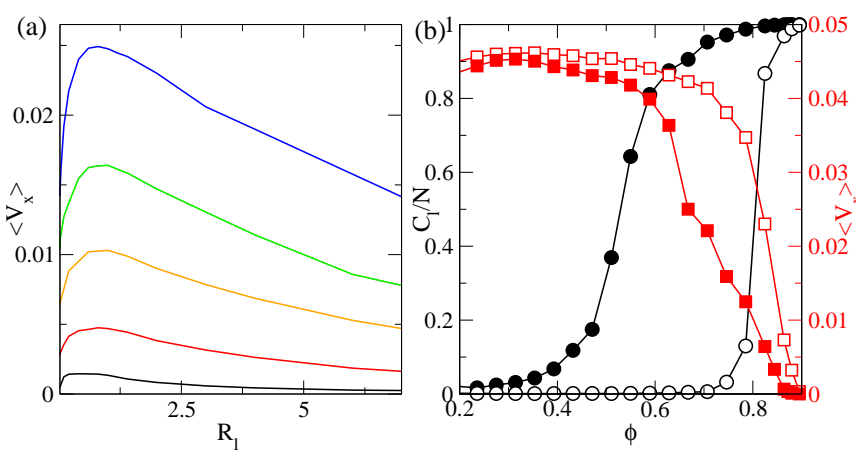

FIG. 4: (a) $\left\langle V_{x}\right\rangle$ vs $R_{l}$ for systems with $\phi_{p}=0.0942$ at $\phi=0.667,0.746,0.785,0.8246$, and 0.864 (from top to bottom) showing the decrease in the transport for increasing $\phi$. (b) $C_{l} / N$ (circles) and $\left\langle V_{x}\right\rangle$ (squares) vs $\phi$ for samples with $\phi_{p}=0.0235$ at $R_{l}=0.04$ (open symbols) and $R_{l}=20$ (filled symbols) showing the correlation between the onset of clustering and the drop in the transport.

onal solid at $\phi=0.9$. Recently it was shown that the addition of quenched disorder to a jamming system decreases the jamming density, where jamming was defined to occur when the drift velocity $\left\langle V_{x}\right\rangle=0$ under an external applied drive [35]. In the active matter system for the non-active limit of $R_{l}=0$, we can obtain a jammed state with $\left\langle V_{x}\right\rangle=0$ by increasing $\phi$ for fixed $\phi_{p}$ or increasing $\phi_{p}$ for fixed $\phi$. In Fig. 4(a) we plot $\left\langle V_{x}\right\rangle$ vs $R_{l}$ for samples with $\phi_{p}=0.0942$ at $\phi=0.667,0.746$, $0.785,0.8246$, and 0.864 . Each curve has an a optimal $R_{l}$ for transport, and the magnitude of $\left\langle V_{x}\right\rangle$ decreases with increasing $\phi$. For $\phi>0.746,\left\langle V_{x}\right\rangle=0$ at $R_{l}=0$, corresponding to the jammed phase. For nonzero $R_{l}$, the decrease in $\left\langle V_{x}\right\rangle$ with increasing $\phi$ is due to the formation of clusters which increase in size with increasing $\phi$. For moderate to large values of $\phi_{p}$, we find that the system reaches the $\left\langle V_{x}\right\rangle=0$ jammed state if infinite run times are used, so that complete jamming can occur at the limits $R_{l}=0$ and $R_{l}=\infty$. In Fig. 4(b) we plot $C_{l} / N$ and $\left\langle V_{x}\right\rangle$ versus $\phi$ for samples with $\phi_{p}=0.0235$ at $R_{l}=0.04$ and $R_{l}=20$. For $R_{l}=0.04,\left\langle V_{x}\right\rangle$ is constant over most of the range of $\phi$ but rapidly decreases to a value near zero at $\phi=0.9$, where we also find a rapid increase in $C_{l} / N$. For $R_{l}=20$, the onset of clustering occurs at a lower value of $\phi$ and is also correlated with a drop in $\left\langle V_{x}\right\rangle$. Our results suggest the possibility of adding a new axis to the jamming phase diagram [33] corresponding to the activity $R_{l}$, where for large activity there could be another critical jamming point $A_{j}$ similar to point J.

We find that increasing the run length or particle density can substantially decrease the mobility of active matter particles due to cluster formation and local clogging. In order to avoid such clogging effects, new types of motion rules could be devised for the active particles, such as having the particles reverse or randomize their swimming direction if they become immobilized by an obstacle or by entrapment in a cluster. It would interesting to explore whether biologically relevant active matter such as swimming organisms have evolved methods to avoid selfclogging. Other types of behavior could arise for different types of obstacles; for example, instead of impenetrable obstacles, the disorder could take the form of sticky sites that reduce the mobility of the particles but that permit their passage. Such pointlike pinning sites would be effective at pinning individual particles, but would have difficulty trapping an extended object such as a cluster.

Summary- We have examined the transport of runand-tumble active matter particles driven through quenched disordered environments. Unlike thermalized particles driven over random disorder, where previous studies have shown that the transport is generally increased when the thermal fluctuations increase, we find that for active matter the transport first increases and then decreases as the activity level or running length increases due to the formation of living crystals that can be locally jammed or clogged. The system becomes effectively jammed at lower densities as the run length increases, suggesting that activity could form a new axis of the jamming phase diagram. Since recent theoretical work has shown that run-and-tumble dynamics and active Brownian motion can be mapped to each other [33], our results can be generalized to other active systems driven over quenched disorder.

We thank L. Lopatina for useful discussions. This work was carried out under the auspices of the NNSA of the U.S. DoE at LANL under Contract No. DE-AC5206NA25396.

[1] S. Ramaswamy, Annu. Rev. Condens. Matter Phys. 1, 323 (2010).

[2] B. ten Hagen, S. van Teeffelwn, and H. Löwen, J. Phys.: Condens. Matter 23, 194119 (2011); H.H. Wensink and H. Löwen, J. Phys.: Condens. Matter 24, 464130 (2012); P. Romanczuk, M. Bär, W. Ebeling, B. Lindner, and L. Schimansky-Geier, Eur. Phys. J. Spec. Top. 202, 1 (2012).

[3] M.C. Marchetti, J.F. Joanny, S. Ramaswamy, T.B. Liverpool, J. Prost, M. Rao, and R.A. Simha, Rev. Mod. Phys. 85, 1143 (2013).

[4] H.C. Berg, Random Walks in Biology (Princeton University Press, Princeton, 1983).

[5] P. Galajda, J. Keymer, P. Chaikin, and R. Austin, J. Bacteriol. 189, 8704 (2007).

[6] T. Vicsek, A. Czirók, E. Ben-Jacob, I. Cohen, and O. Shochet, Phys. Rev. Lett. 75, 1226 (1995); H. Chaté, F. Ginelli, G. Grégoire, F. Peruani, and F. Raynaud, Eur. Phys. J. B 64, 451 (2008).

[7] W.F. Paxton, K.C. Kistler, C.C. Olmeda, A. Sen, S.K. St. Angelo, Y.Y. Cao, T.E. Mallouk, P.E. Lammert, and V.H. Crespi, J. Am. Chem. Soc. 126, 13424 (2004); J.R. Howse, R.A.L. Jones, A.J. Ryan, T. Gough, R. Vafabakhsh, and R. Golestanian, Phys. Rev. Lett. 99, 
048102 (2007).

[8] R. Golestanian, Phys. Rev. Lett. 102, 188305 (2009).

[9] G. Volpe, I. Buttinoni, D. Vogt, H.-J. Kummerer, and C. Bechinger, Soft Matter 7, 8810 (2011).

[10] J. Palacci, S. Sacanna, A.P. Steinberg, D.J. Pine, and P.M. Chaikin, Science 339, 936 (2013).

[11] R. Dreyfus, J. Baudry, M.L. Roper, M. Fermigier, H.A. Stone, and J. Bibette, Nature (London) 437, 862 (2005).

[12] L. Giomi, N. Hawley-Weld, and L. Mahadevan, Proc. Roy. Soc. A 469, 20120637 (2013).

[13] J. Bialké, T. Speck, and H. Löwen, Phys. Rev. Lett. 108, 168301 (2012).

[14] S. Henkes, Y. Fily, and M. C. Marchetti, Phys. Rev. E 84, 040301 (2011); Y. Fily and M.C. Marchetti, Phys. Rev. Lett. 108, 235702 (2012).

[15] M.E. Cates and J. Tailleur, EPL 101, 20010 (2013).

[16] I. Theurkauff, C. Cottin-Bizonne, J. Palacci, C. Ybert, and L. Bocquet, Phys. Rev. Lett. 108, 268303 (2012).

[17] G.S. Redner, M.F. Hagan, and A. Baskaran, Phys. Rev. Lett. 110, 055701 (2013).

[18] I. Buttinoni, J. Bialké, F. Kümmel, H. Löwen, C. Bechinger, and T. Speck, Phys. Rev. Lett. 110, 238301 (2013).

[19] J. Stenhammar, A. Tiribocchi, R.J. Allen, D. Marenduzzo, and M.E. Cates, Phys. Rev. Lett. 111, 145702 (2013).

[20] B.M. Mognetti, A. Saric, S. Angioletti-Uberti, A. Cacciuto, C. Valeriani, and D. Frenkel, Phys. Rev. Lett. 111, 245702 (2013).

[21] M.B. Wan, C.J. Olson Reichhardt, Z. Nussinov, and C. Reichhardt, Phys. Rev. Lett. 101, 018102 (2008); C. Reichhardt and C.J. Olson Reichhardt, Phys. Rev. E 88, 062310 (2013).

[22] J. Tailleur and M.E. Cates, Europhys. Lett. 86, 60002 (2009).

[23] J.A. Drocco, C.J. Olson Reichhardt, and C. Reichhardt, Phys. Rev. E 85, 056102 (2012).

[24] M.E. Cates, Rep. Prog. Phys. 75, 042601 (2012).

[25] L. Angelani, A. Costanzo and R. Di Leonardo, EPL 96, 68002 (2011); V. Kantsler, J. Dunkel, M. Polin, and R.E. Goldstein, Proc. Natl. Acad. Sci. USA 110, 1187 (2013); I. Berdakin, Y. Jeyaram, V.V. Moshchalkov, L. Venken, S. Dierckx, S.J. Vanderleyden, A.V. Silhanek, C.A. Condat, and V.I. Marconi, Phys. Rev. E 87, 052702 (2013).

[26] O. Chepizhko, E.G. Altmann, and F. Peruani, Phys. Rev. Lett. 110, 238101 (2013).

[27] O. Chepizhko and F. Peruani, Phys. Rev. Lett. 111, 160604 (2013).

[28] C. Reichhardt and C.J. Olson, Phys. Rev. Lett. 89, 078301 (2002); A. Pertsinidis and X.S. Ling, Phys. Rev. Lett. 100, 028303 (2008); P. Tierno, Phys. Rev. Lett. 109, 198304 (2012).

[29] G. Blatter, M.V. Feigelman, V.B. Geshkenbein, A.I. Larkin, and V.M. Vinokur, Rev. Mod. Phys. 66, 1125 (1994).

[30] G. Grüner, Rev. Mod. Phys. 60, 1129 (1988).

[31] F.I.B. Williams, P.A. Wright, R.G. Clark, E.Y. Andrei, G. Deville, D.C. Glattli, O. Probst, B. Etienne, C. Dorin, C.T. Foxon, and J.J. Harris, Phys. Rev. Lett. 66, 3285 (1991); H.-W. Jiang, H.L. Stormer, D.C. Tsui, L.N. Pfeiffer, and K.W. West, Phys. Rev. B 44, 8107 (1991).

[32] S. Lemerle, J. Ferré, C. Chappert, V. Mathet, T. Giamarchi, and P. Le Doussal, Phys. Rev. Lett. 80, 849 (1998); P.J. Metaxas, J.P. Jamet, A. Mougin, M.
Cormier, J. Ferré, V. Baltz, B. Rodmacq, B. Dieny, and R.L. Stamps, Phys. Rev. Lett. 99, 217208 (2007).

[33] A.J. Liu and S.R. Nagel, Nature (London) 396, 21 (1998).

[34] C.S. OHern, L.E. Silbert, A.J. Liu, and S.R. Nagel, Phys. Rev. E 68, 011306 (2003); J.A. Drocco, M.B. Hastings, C.J. Olson Reichhardt, and C. Reichhardt, Phys. Rev. Lett. 95, 088001 (2005).

[35] C.J. Olson Reichhardt, E. Groopman, Z. Nussinov, and C. Reichhardt, Phys. Rev. E 86, 061301 (2012).

[36] S. Luding and H.J. Herrmann, Chaos 9, 673 (1999).

[37] See supplemental information. 
SUPPLEMENTARY MATERIAL FOR ACTIVE MATTER TRANSPORT AND JAMMING ON DISORDERED LANDSCAPES

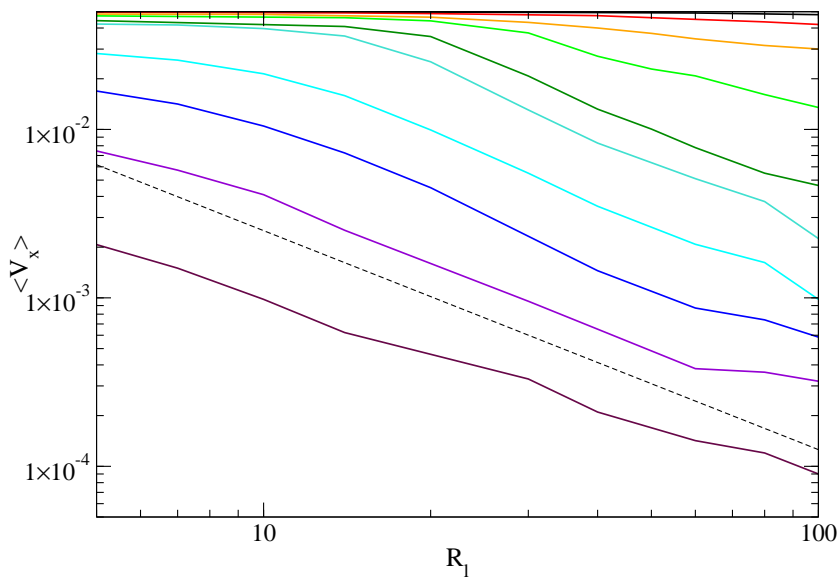

FIG. 1: A log-log plot of $\left\langle V_{x}\right\rangle$ vs $R_{l}$ for systems with $\phi=0.667$ and $F_{d}=0.05$ at $\phi_{p}=0.00039,0.00157,0.00472$, $0.0785,0.0157,0.02356,0.055,0.0942,0.01413$, and 0.188 , from top to bottom. The dashed line is a fit to a power law with $V_{x}\left(R_{l}\right) \propto R_{l}^{\alpha}$, with $\alpha=1.31 \pm 0.02$. 


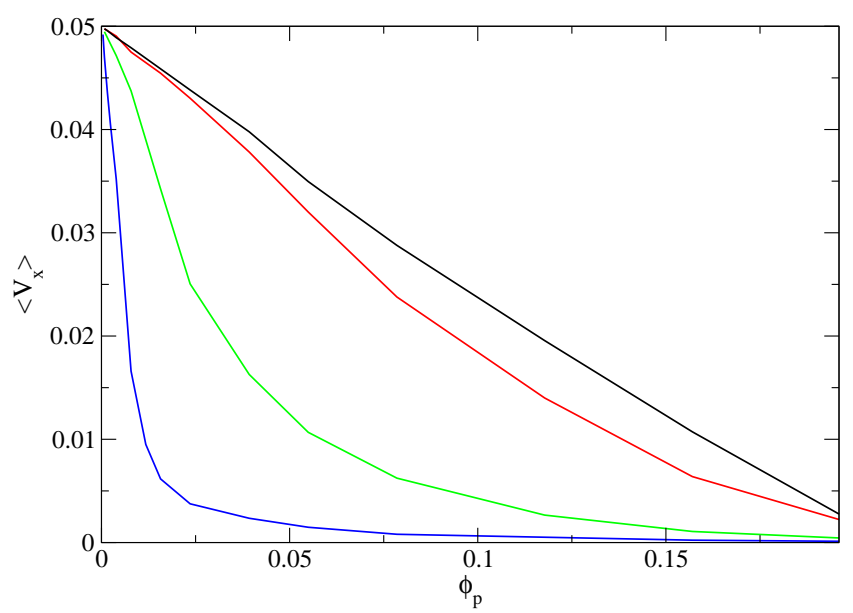

FIG. 2: $\left\langle V_{x}\right\rangle$ vs $\phi_{p}$ at a fixed $\phi=0.67$ for $R_{l}=1.0,4.0$, 20.0 , and 80.0 , from top to bottom. At small run lengths, $\left\langle V_{x}\right\rangle$ decreases linearly with increasing obstacle density, while for large run lengths the decrease is much more rapid and approximately follows $\left\langle V_{x}\right\rangle \propto 1 / \phi_{p}$. 